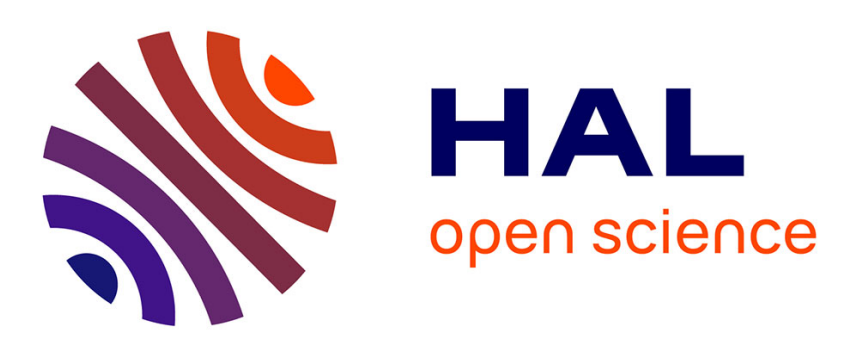

\title{
An improved semi-analytical solution for stress at round-tip notches
}

Mingchao Liu, Yixiang Gan, Dorian A.H. Hanaor, Bin Liu, Chang-Qing Chen

\section{To cite this version:}

Mingchao Liu, Yixiang Gan, Dorian A.H. Hanaor, Bin Liu, Chang-Qing Chen. An improved semianalytical solution for stress at round-tip notches. Engineering Fracture Mechanics, 2015, 149, pp.134143. 10.1016/j.engfracmech.2015.10.004 . hal-02310717

\section{HAL Id: hal-02310717 https://hal.science/hal-02310717}

Submitted on 5 Nov 2019

HAL is a multi-disciplinary open access archive for the deposit and dissemination of scientific research documents, whether they are published or not. The documents may come from teaching and research institutions in France or abroad, or from public or private research centers.
L'archive ouverte pluridisciplinaire HAL, est destinée au dépôt et à la diffusion de documents scientifiques de niveau recherche, publiés ou non, émanant des établissements d'enseignement et de recherche français ou étrangers, des laboratoires publics ou privés. 


\title{
An improved semi-analytical solution for stress at round-tip
}

\section{notches}

\author{
Mingchao Liu ${ }^{1,2}$, Yixiang Gan ${ }^{2,}$, Dorian A.H. Hanaor ${ }^{2}$, Bin Liu ${ }^{1}$, and Chang- \\ Qing Chen ${ }^{1, *}$
}

1. Department of Engineering Mechanics, CNMM \& AML, Tsinghua University, Beijing 100084, China

2. School of Civil Engineering, The University of Sydney, Sydney, NSW 2006, Australia

\begin{abstract}
In order to investigate the brittle failure of key-hole-notched components, the stress distribution at notch tips is studied numerically and theoretically. A semi-analytical formula is developed for the maximum notch-tip-stress, incorporating crack-tip-blunting, stress-concentration and stress-equilibrium. Stress distributions in notched plates are simulated by finite-element method showing improved accuracy of the formula relative to established solutions. Application of the developed equation to components containing U-notches and blunt V-notches, is also explored, demonstrating its broad applicability. When combined with stress-based failure criteria, the semi-analytical model can be employed to assess brittle failure in notched components with significance towards fracture in heterogeneous materials.
\end{abstract}

Keywords: Key-hole notches; Crack tip blunting; Stress concentration; Stress equilibrium; Failure criterion

\section{Introduction}

As widely observed in engineering structures and experimental specimens, the existence of notches often results in inhomogeneous stress distribution and leads to the failure of components [1-4]. Common notch geometries include key-hole notches [5], i.e. a slit ending with a circular hole, and types of key-hole-like notches including U-notches [6] and blunt Vnotches ( V-notches with rounded tips [7] or end holes [8]). Rather than being solely a defect, carefully introduced notches can be used to alleviate stress concentration at the tip of cracks or

\footnotetext{
*Corresponding authors. Emails: yixiang.gan@sydney.edu.au (Y. Gan) and chencq@tsinghua.edu.cn (C.Q. Chen).
} 
slits. A common example is the repair of structural components damaged by small cracks by drilling a hole at the crack tip to reduce the stress concentration. With such methods, a slit ending in a circular hole is obtained, which can be considered as a key-hole notch [9, 10]. Due to their frequent occurrence in engineering structures, understanding the stress distribution and failure criteria of notched components is of significant importance in damage resistance evaluation [11].

Numerous studies have been conducted to investigate the brittle failure of notched components with either key-hole or key-hole-like notches. The analysis of failure in defected structures under various loading conditions constitutes a significant aspect of classic fracture mechanics and theoretical frameworks have been established to provide a reliable basis for assessing engineering design and structural safety [12]. For notched components, the stress singularity of cracks is substituted by a region of high stress concentrated at the tip, which is correlated to the notch geometry. The field of notch fracture mechanics (NFM), which is an extension of the classic fracture mechanics of cracked domains, has been developed by introducing the notch stress intensity factor (NSIF, denoted as $K_{\mathrm{I}}^{*}$ ) and notch fracture toughness (NTF, denoted as

$K_{\mathrm{Ic}}^{*}$ ) [13]. There are several stress-based criteria and theories in the context of NFM for the assessment of brittle failure in notched components under mode I loading. These theories include the cohesive zone model (CZM) [14], finite fracture mechanics (FFM) [15], point-stress (PS) and mean-stress (MS) criteria [7, 16], and the maximum hoop stress criterion [17], among others. All these criteria predict brittle fracture on the basis of a simple closed-form solution whereby $K_{\mathrm{I}}^{*}=K_{\mathrm{Ic}}^{*}$, which is similar to Irwin's model in classic fracture mechanics. It should be noted that the maximum hoop stress criterion is believed to be more suitable for crack kinking in notched components of brittle materials, even under mode I loading.

The application of failure criteria in notched components necessitates the development of improved methods for stress analysis. Based upon an earlier work of Creager and Paris [18], Kullmer and Richard [19] presented an asymptotical elastic stress distribution around a keyhole notch tip by introducing the concept of effective crack length. They developed a brittle fracture criterion based on the estimated stress field and considered the notch fracture toughness $K_{\mathrm{I} c}^{*}$ as a generalization of the crack toughness $K_{\mathrm{I} c}$, where $K_{\mathrm{I} c}$ forms the lower bound of $K_{\mathrm{Ic}}^{*}$. It is important to note that since the asymptotical stress field was deduced from Airy's stress function in their work, the solution is only valid when the radius of key-hole is much smaller than the crack length. In recent years, on the basis of the work of Kullmer and Richard, NFM has been broadly applied to the evaluation of failure resistance in key-hole notches $[5,9$, $10,20]$. Stress fields of two typical examples of key-hole-like notches (U-notches and blunt Vnotches) have been studied [21-24]. The widely used stress field solution of round-tip V-notches given by Filippi et al. [21] has been shown to agree well with the finite element results in the 
high stress region around the notch tip. Subsequently, this stress field prediction has been extended to the case of finite size plates [22]. Recently, the stress field solution of key-hole notches given by Kullmer and Richard has been extended to geometries consisting of V-notches with end holes [23] and blunt cracks in anisotropic plates under in-plane loadings [24]. It should be noted that the maximum principal notch tip stress was introduced into the expression of stress components as an unknown parameter, and, subsequently, the NSIF was related to the maximum stress [21, 22]. It can thus be seen that an effective prediction of the maximum stress at notch tips is important for the application of the stress field solutions and their corresponding fracture criteria.

For typical heterogeneous materials (e.g. rocks and ceramics), the inevitable presence of voids around crack tips has significant effect on their fracture properties. Bazant discovered that crack tips in concrete and rock are blunted by the existing of micro pores, and similar effects were found in ductile metals through the presence of plastic zones [25]. Smith showed that the role of crack tip pores could not be ignored, regardless of size, giving rise to the so-called "key-hole problem" [26]. Further studies have indicated that, when pores exist at crack tips, there exist competing effects of nominal toughness enhancement due to crack blunting by pores and the weakening caused by the increasing volume fraction of pores [27]. As a consequence, recently established approaches for modelling key-hole notches can be applied to fracture analysis of porous materials [28].

In the present work, the stress distribution of a notched finite rectangular plate under remote uniaxial tensile stress is studied. In Section 2, a semi-analytical formula, which is based on the combined actions of crack tip blunting, stress concentration and stress equilibrium, is presented to predict the maximum notch tip stress. Feasibility of the prediction is discussed in view of FEM results in Section 3. FEM simulations of the stress distributions of U-notches and blunt V-notches are shown in Section 4, demonstrating that the equation presented for key-hole notches is applicable also to U-notches and blunt V-notches with large notch tip radii and small open-angles. The paper is finished with a few conclusions in the last section.

\section{Stress analysis of key-hole notched components}

The presence of holes, cracks and notches complicates the prediction of stress fields in structures. For components with cracks, the maximum stress cannot be defined due to the stress singularity at the crack tip. Irwin introduced the concept of stress intensity factor (SIF) to characterize the extent of stress singularity at crack tip, which forms the foundation of linear elastic fracture mechanics (LEFM) [29]. In order to calculate the SIF of different type of cracks, a comprehensive description of the weight function technique has been given by Fett and Munz [30]. For structures with voids around the crack tip, the singularity can be reduced or even removed, owing to the blunting effect induced by crack tip microstructures [29, 30], but nonetheless defect localized stresses still exist. In the failure of structures, various mechanisms can prevail: For crack or crack-like defects under mode I loading, the normal stress perpendicular to the crack plays a leading role in the failure of structures [33]; in fatigue life predictions of notched components, only the maximum principal stress is generally taken into 
Liu, M., Gan, Y., Hanaor, D. A., Liu, B., \& Chen, C. (2015). An improved semi-analytical solution for stress at round-tip notches. Engineering fracture mechanics, 149, 134-143.

account $[21,22]$. Therefore, stress analysis is of paramount important for notched components. In the following, such an analysis is performed for the mode I loading of structural components containing notch defects.

\subsection{Stress fields of defected plates}

To investigate the stress distributions of defected components, the stress distributions of a simple defected structural component - a two dimensional finite rectangular plate with an edge crack as shown in Fig. 1(a) by the black solid lines is first considered, where $\sigma_{\infty}$ is the remote loading in the $y$ direction, $a$ is the crack length, $w$ is the plate width, and $b$ is the plate height. At the same time, a finite plate with a key-hole notch is also shown in Fig. 1(a) by red dotted line with the same loading condition. The center point is located at the crack tip and radius is denoted $\rho$. The numerical solutions for the tip stress field of defected structures containing a standard crack and a key-hole notch are shown in Fig. 1(b), using $a / w=0.4$ and $\rho=0.05$ as a typical example providing the $y$-direction normal stress distribution along the crack or notch bisector $(y=0$ and $x>0)$.
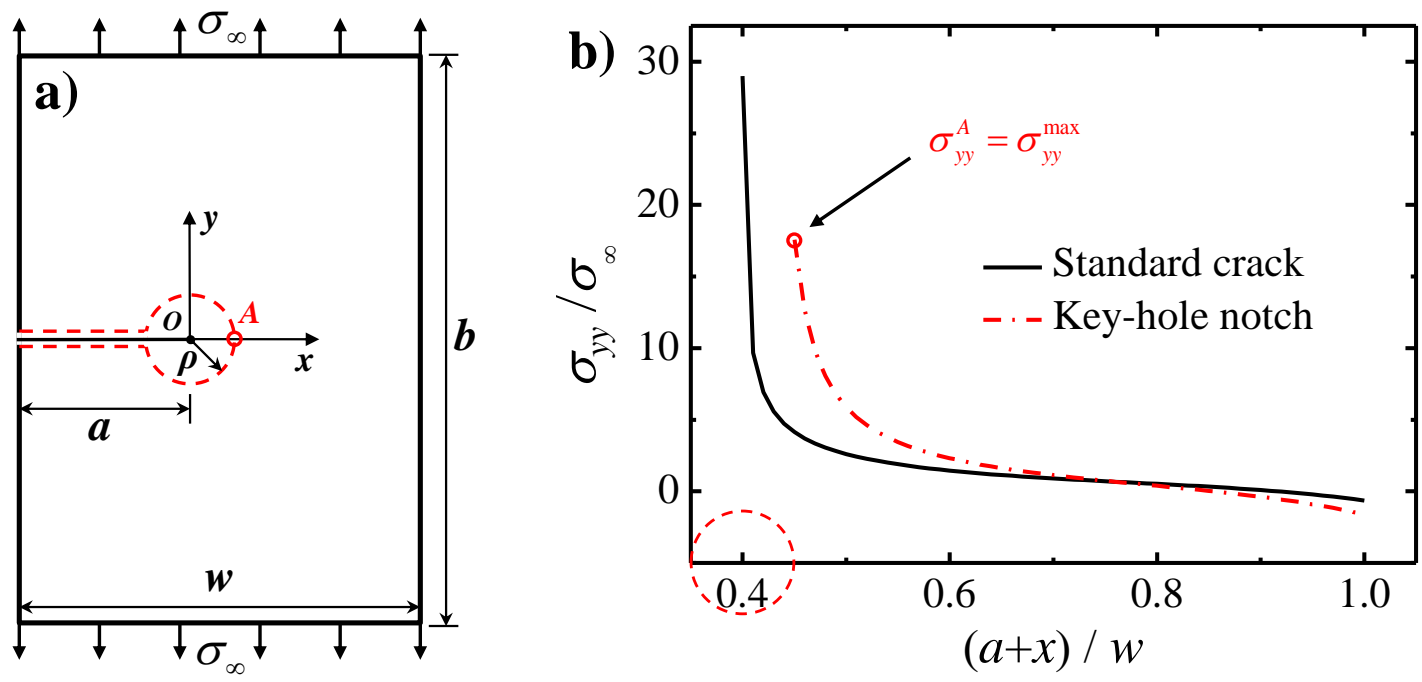

Fig. 1 (a) Standard crack (black solid line) and Key-hole notch (red dashed line) together with their Cartesian coordinate system; (b) The tip stress distributions along the bisector for the crack (black solid line) and notch (red dashed line).

According to LEFM, the normal stress $\sigma_{y y}$ along the crack line of the problem shown in Fig.

1(a) under mode I loading is related to $K_{\mathrm{I}}$ (SIF) by 


$$
\sigma_{y y}=\frac{K_{\mathrm{I}}}{\sqrt{2 \pi x}}, x>0
$$

where $K_{\mathrm{I}}$ is given by

$$
K_{\mathrm{I}}=\sigma_{\infty} \cdot \sqrt{\pi a} \cdot f(\eta), \quad \eta=a / w
$$

Here, $f(\eta)$ is the geometric shape factor for unconstrained specimens and is given by [34]

$$
f(\eta)=1.12-0.23 \eta+10.56 \eta^{2}-21.74 \eta^{3}+30.42 \eta^{4}
$$

According to Ref. [34], the error of Eq. (3) is less than $0.5 \%$ for $b / w \geq 1$ and $a / w \leq 0.6$.

Eq. (1) is the asymptotic solution of the crack tip stress field, which is accurate only near the crack tip. It is noted that, for a crack in an infinitely large plate, an exact solution is available for the stress field, which is valid not only at the crack tip but also for the far field. The corresponding normal stress along the crack line has the form of $[35,36]$,

$$
\sigma_{y y}=\frac{K_{\mathrm{I}} \cdot(x+a)}{\sqrt{\pi a} \cdot \sqrt{x^{2}+2 a x}}, x>0,
$$

where $K_{\mathrm{I}}$ is the stress intensity factor. In this study, we extended Eq. (4) to finite width plates

with edge cracks by assuming $K_{\mathrm{I}}$ has the same form as Eq. (2).

For key-hole notches (i.e., cracks having a hole at their tip as represented in Fig. 1(a) by the red dashed line), the stress singularity can be removed due to the existence of the hole. Kullmer and Richard [19] obtained closed-form solution for the asymptotical elastic stress distribution around a central slit ending in a circular hole in an infinite large disk. According to their solution, the $y$-direction normal stress component along the notch bisector can be expressed as

$$
\sigma_{y y}=\frac{K_{\mathrm{I}}^{\prime}}{4 \sqrt{2 \pi x}}\left[4+\frac{5}{2} \frac{\rho}{x}+3\left(\frac{\rho}{x}\right)^{2}+\frac{5}{2}\left(\frac{\rho}{x}\right)^{3}\right], x \geq \rho .
$$

Considering the introduced effective crack length, $a^{\prime}=a+\rho$, the corresponding effective SIF for components with finite geometry can be assumed to have the following form

$$
K_{\mathrm{I}}^{\prime}=\sigma_{\infty} \cdot \sqrt{\pi(a+\rho)} \cdot f(\eta) .
$$

It can be seen from Eq. (5) that the normal stress component $\sigma_{y y}$ decreases with increasing distance from the edge of the notch, reaching its maximum value at the notch tip, that is,

$$
\sigma_{y y}^{\max }=\left.\sigma_{y y}\right|_{x=\rho}=\frac{3 K_{\mathrm{I}}^{\prime}}{\sqrt{2 \pi \rho}}=3 \sigma_{\infty} \cdot \sqrt{\frac{a+\rho}{2 \rho}} \cdot f(\eta) .
$$

Note that Eq. (5) is based upon the assumption that the radius of the crack-tip-hole is small 
compared to the crack length (e.g., $\rho / a<0.1$ ). For plates with a large tip-hole relative to the crack length, there is yet no established formula to reliably predict the maximum stress. In the following section, a semi-analytical formula to predict the maximum stress around key-hole notch tips will be developed.

\subsection{Prediction of notch tip maximum stress}

For the key-hole notched component shown in Fig. 1(a), three major factors affecting the notch tip stress distribution. First, the crack induced stress can be estimated in accordance with Eq. (4). The presence of the hole at the crack tip results in crack blunting, thus removing the stress singularity. As crack tip stress singularity is related to crack length, the key-hole induced blunting effect corresponds to the crack length. Secondly, as a result of the limited plate width, $w$, compared to the standard crack with length $a$ (effective bearing width of $w-a$ ), the effective load bearing area along the crack bisector $y=0$ is reduced to $w-a-\rho$ when there exists a round hole with radius $\rho$ at the crack tip. The stress equilibrium factor can be derived from the force and torque balance by comparing stress fields in cases for cracks with and without the hole using corresponding linear approximations of the normal stress along the crack bisector. Thirdly, for a finite rectangular plate significant stress concentration occurs around the circular hole. Taking above three majors effects into account, a semi-analytical model to predict the notch tip maximum stress can be assumed to be

$$
\sigma_{y y}^{\max }=\frac{\sigma_{\infty} \cdot(a+\rho)}{\sqrt{\rho^{2}+2 a \rho}} \cdot f(\eta) \cdot K_{s} \cdot K_{t},
$$

where $K_{s}$ denotes the stress equilibrium factor based on the force and torque balance effect, and it can be obtained as

$$
K_{s}=\left(\frac{w-a}{w-a-\rho}\right)^{2} .
$$

The stress concentration factor $K_{t}$ of a circular hole in a finite plate subject to uniaxial tension can be approximated by [37]

$$
K_{t}=2\left(1-\frac{2 \rho}{w}\right)^{-1}+\left(1-\frac{2 \rho}{w}\right)^{2},
$$

which increases from 3 monotonically with $\rho / w$. It can be seen from Eq. (8) that the maximum stress at the notch tip, $\sigma_{y y}^{\max }$, is related to geometrical parameters (plate width $w$, crack length $a$, and key-hole radius $\rho$ ) and loading conditions ( remote tensile stress $\sigma_{\infty}$ ). However, it is worth noting that the aspect ratio $b / w$ of the finite rectangular plate has a significant impact 
on the stress concentration factor only when it is less than 2 .

For a semi-edge notch in a semi-infinite rectangular plate, which is common in engineering problems, with plate width $w \rightarrow \infty, f(\eta) \rightarrow 1.12$ should be substituted into Eq. (7) and we obtain

$$
\sigma_{y y}^{\max }=3.36 \sigma_{\infty} \cdot \sqrt{\frac{a+\rho}{2 \rho}}
$$

Whereas substituting $f(\eta) \rightarrow 1.12, K_{s} \rightarrow 1$ and $K_{t} \rightarrow 1$ into Eq. (8) for the case of semi-infinite plate yields

$$
\sigma_{y y}^{\max }=3.36 \sigma_{\infty} \cdot \frac{(a+\rho)}{\sqrt{\rho^{2}+2 a \rho}} .
$$

It can be seen that for the special case of semi-infinite plates, our model (Eq. (12)) and the Kullmer-Richard model (Eq. (11)) predict similar results of the maximum stress, for small values of $\rho / a$. For large values of $\rho / a$, however, the Kullmer-Richard model differs significantly from our model, as illustrated in Fig. 3 and detailed in Section 3.2.

\section{Finite element simulations}

In order to validate the proposed semi-analytical solution (8), notch tip stress fields were calculated numerically using the commercial finite element software package ANSYS12.0. The plate of the model is meshed with 8-node biquadratic plane strain elements (Solid Quad 8Node 82 elements). Due to symmetry, only half of the model $(y>0)$ in Fig. 1(a) is meshed and symmetrical boundary conditions are enforced along the notch bisector $(y=0$ and $x>0$ ).

The adopted loading conditions are remote tensile stress $\sigma_{\infty}$ at the edge $y=b / 2$ shown in Fig. 1(a), with the stress free boundary conditions employed for the rest boundary edges. A linear elastic material with Young's modulus of $E=70 \mathrm{GPa}$ and Poisson ratio $v=0.3$ was used with mesh sensitivity analyses conducted to ensure the numerical convergence of the model. Considering the concentrated stress around the notch tip, graded meshes are employed for the tip region.

\subsection{Effects of aspect ratio $b / w$}

In order to clarify the geometrical influence on the numerical simulations, several groups of key-hole notched plates with the same notch size (crack length $a$ and tip curvature $\rho$ ) and different aspect ratios $(b / w)$ were studied by FEM. All the cases are under remote uniform 
Liu, M., Gan, Y., Hanaor, D. A., Liu, B., \& Chen, C. (2015). An improved semi-analytical solution for stress at round-tip notches. Engineering fracture mechanics, 149, 134-143.

tension in the $y$-direction, as shown in Fig. 2(a). The stress distribution and corresponding stress concentration (or singularity) are related to the geometry of finite rectangular plates containing defects. The FEM results overall showed similar stress distributions with notable differences in the high stress region and the maximum stress. It was further found that the maximum stress level changes significantly with increasing aspect ratio, following the relationship shown in Fig. 2. For short crack lengths with small hole radii the maximum stress was found to decrease before reaching a convergence value denoted here as $\sigma_{\mathrm{cv}}$ as shown in Fig. 2(a). For long cracks with large holes, the maximum stress was found to increase before converging to $\sigma_{\mathrm{cv}}$ as shown in Fig. 2(b). To determine the aspect ratio regime that shows negligible influence on the resulting maximum stress, the results of plates with different sizes of notches are compared. The maximum stresses normalized by the converging value are shown in Fig. 2(c).
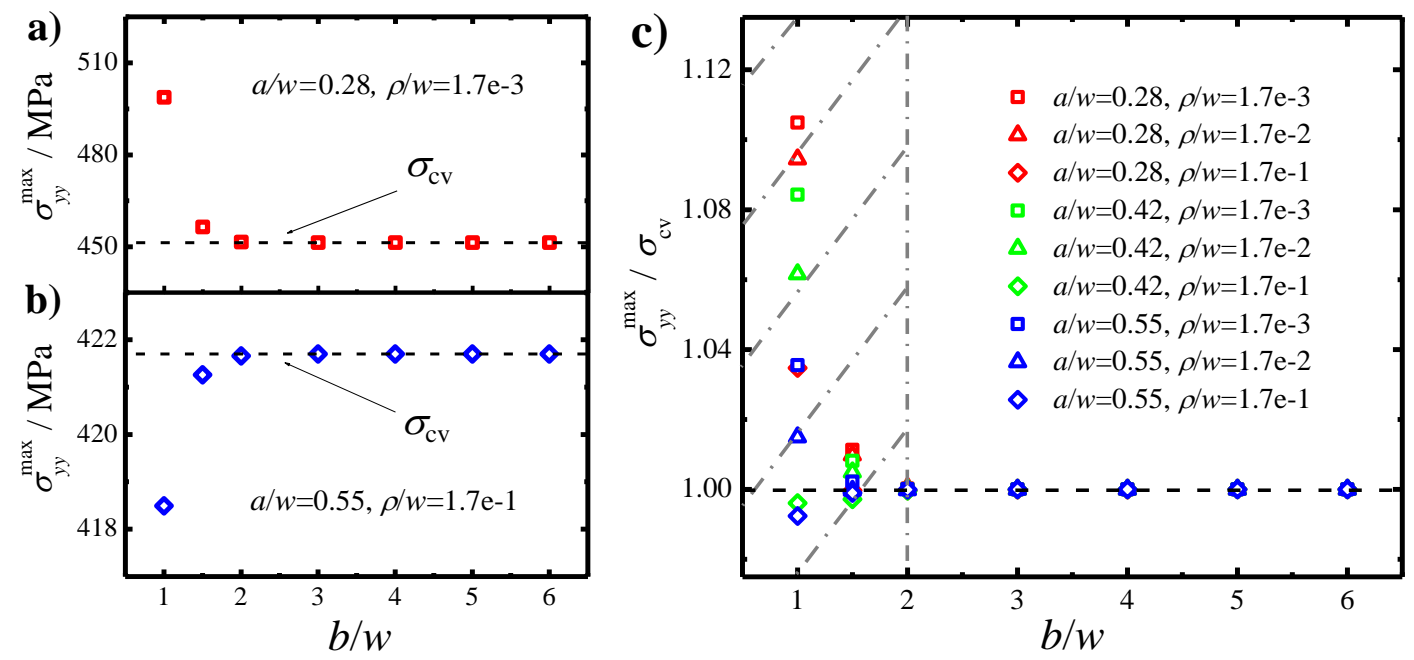

Fig. 2 Notch-tip maximum stress as a function of plate aspect ratio, $b / w$, for different notch geometries: (a) the relative crack length, $a / w=0.28$, and the relative radius of key-hole, $\rho / w=0.0017 ;$ (b) $a / w=0.55$, and $\rho / w=0.17$. (c) Normalized maximum stress varies with the aspect ratio.

Figure 2(c) clearly shows that the notch tip maximum stress gradually decreases or increases with increasing aspect ratio $(b / w)$ in the interval from 1 to 2 for plates with different notch sizes. For aspect ratios satisfying $b / w \geq 2$, the maximum stress converges to stable values for all cases considered. As conditions of $b / w<2$ are of little significance in engineering applications, the divergence of stress vales in this regime is not studied further in the present work. To eliminate geometrical effects from the numerical simulations, all cases discussed subsequently satisfy $b / w \geq 2$ and are thus within the regime of convergent maximum stress. 


\subsection{Numerical results}

For the key-hole notch problem with $a / w=0.42$ shown in Fig. 1(a), the maximum notch tip stress component $\sigma_{y y}$ predicted by the proposed semi-analytical formula (8), the asymptotical solution by Kullmer and Richard [19], and the FEM simulation is shown in Fig. 3(a) as a function of the relative notch tip curvature $\rho / a$. One can see from Fig. 3(a) that the semi-analytical prediction (8) agrees well with the finite element results even for the notch size as big as $\rho / a \approx 0.4$. In contrast, the Kullmer-Richard asymptotical prediction is valid only for very small notches satisfying $\rho / a<0.1$, which is consistent with the assumption of small crack tip curvature adopted by Kullmer and Richard for deriving their results. To be more specific, the relative errors of the semi-analytical prediction and the Kullmer-Richard asymptotical prediction with respect to the finite element results are shown as a function of keyhole radius in Fig. 3(b). It can be seen that the relative error of Eq. (8) is less than $10 \%$ for the hole radius in the range of $\rho / a \leq 0.4$ and can be less than $2 \%$ for $\rho / a \leq 0.25$ However, the relative error from Kullmer-Richard equation is less than $10 \%$ only for $\rho / a \leq 0.05$ and even reaches $60 \%$ at $\rho / a=0.4$. Thus it can be concluded that our proposed semi-analytical formulae can effectively predict the maximum stress at the notch tip for a wider range of notches than the asymptotical equation of Kullmer and Richard.

As a limiting case, notches in semi-infinite plates $(w \rightarrow \infty)$ were also investigated. The maximum stress at the notch tip as a function of the relative hole radius is plotted in Fig. 3(c). The prediction of the Kullmer-Richard equation is also included for the purpose of comparison. Similar to the results of finite plates (Fig. 3(a)), it can be seen that the agreement between the theoretical predictions given by our proposed semi-analytical model and the finite element results is closer than that of asymptotical predictions from the Kullmer-Richard equation. The corresponding relative error of our semi-analytical predictions and the asymptotical predictions given by Kullmer and Richard with respect to finite element results are shown as a function of key-hole radius in Fig. 3(d). The error over the entire range $(\rho / a \leq 1)$ is less than $5 \%$ for our semi-analytical predictions, while the relative error from the Kullmer-Richard equation is less than $5 \%$ only in the range of $\rho / a \leq 0.06$, indicating the developed model (Eq. (12)) can be applied for a much wider range of $\rho / a$ compared to the Kullmer-Richard model (Eq. (11)). 
a)
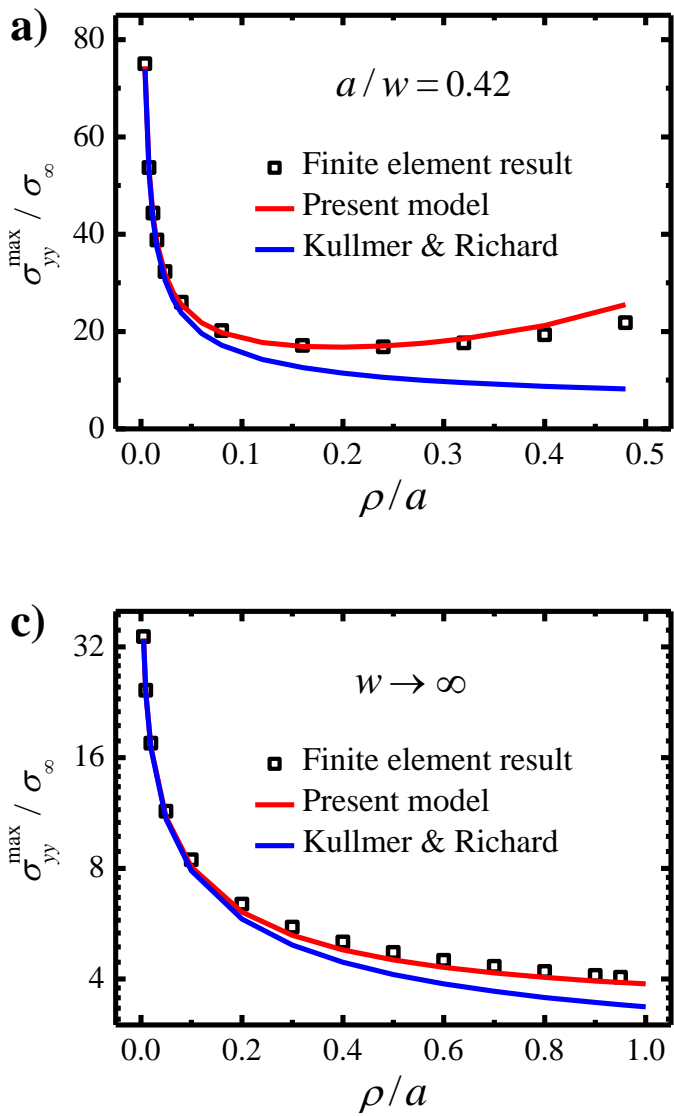
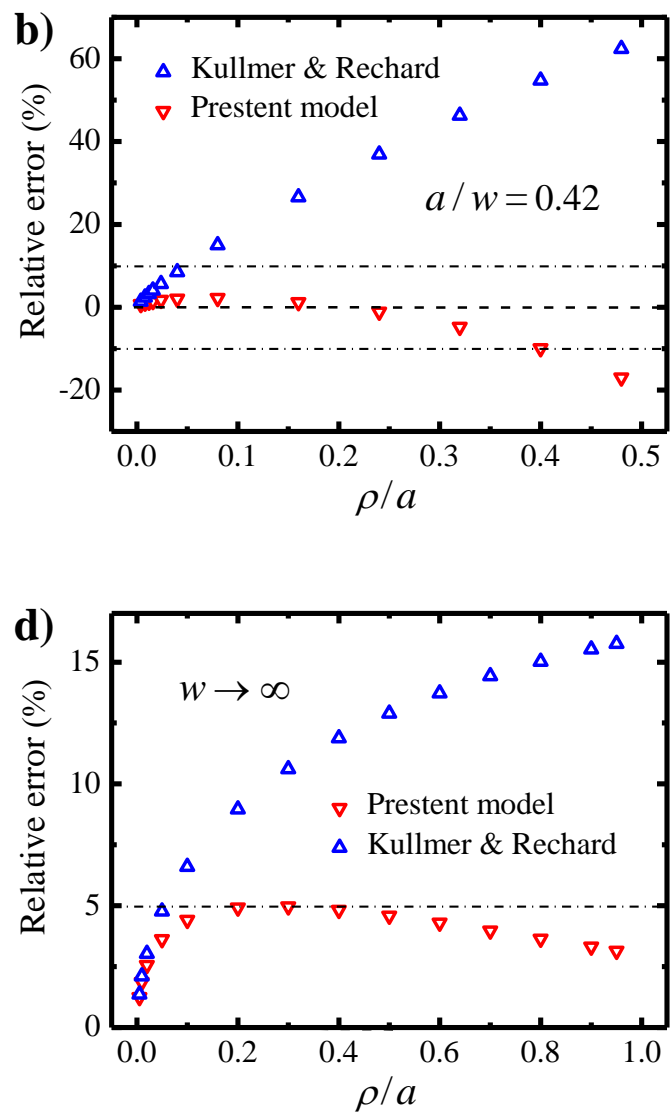

Fig. 3 (a) Normalized maximum stress as a function of the relative key-hole radius. (b) The relative error with respect to FEM results as a function of relative key-hole radius. (c) and (d) respectively show the normalized maximum stress and the relative error for the case of a semiinfinite plate.

Recall that the semi-analytical equation is based on the assumption that three mechanisms of crack tip bluntness, stress concentration and stress equilibrium exhibit an interplay at the notch tip. To further validate of the assumption, the variations of maximum stress at the notch tip with increasing key-hole radius, for cases of fixed crack length ( $a=$ const $)$ and fixed notch depth ( $a+\rho=$ const ), are plotted respectively in Figs. 4(a) and 4(b). Again, the maximum stresses predicted by the semi-analytical equation and the FEM simulations are in good agreement. 

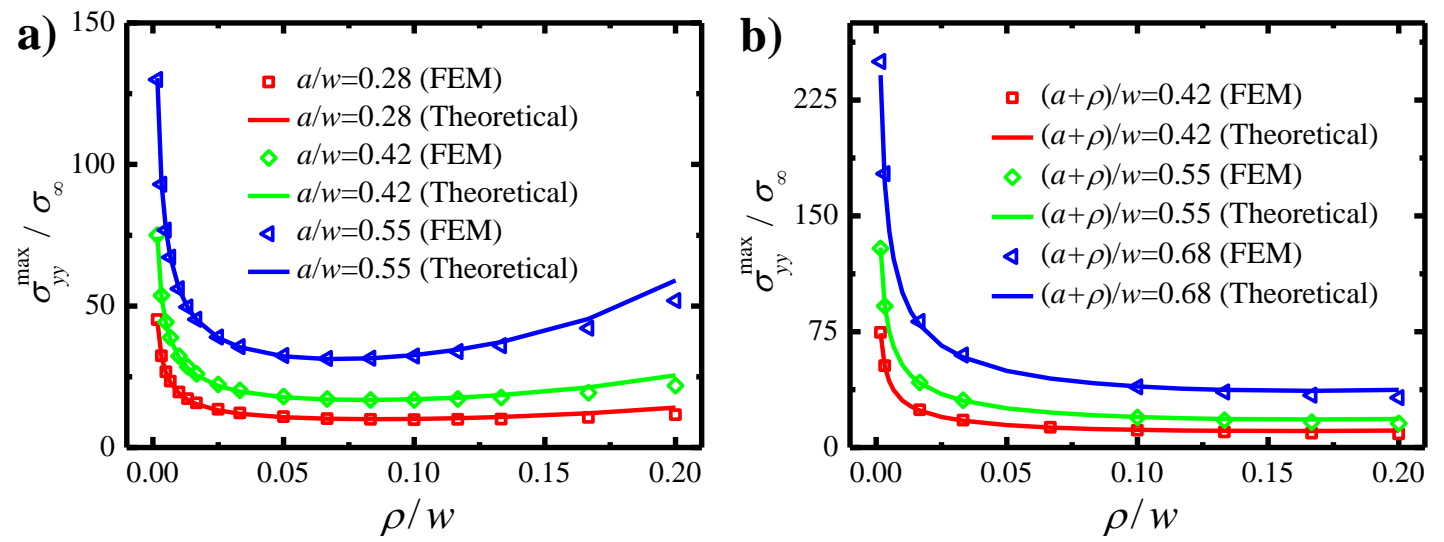

Fig. 4 The relationship between the dimensionless maximum stress and the relative key-hole radius: (a) Fixed crack length $(a=$ const); (b) Fixed notch depth $(a+\rho=$ const).

For fixed crack length values ( $a=$ const ), Fig. 4(a) clearly shows the competition of the three aforementioned mechanisms. As a result, the maximum stress decreases first and then increases with increasing key-hole size. This non-monotonic trend is more obvious for longer cracks. For a fixed key-hole radius, the resulting maximum stress increases with crack length. Longer cracks result in a smaller effective bearing area, and so the maximum notch tip stresses are larger. However, for cases with fixed notch depth $(a+\rho=$ const $)$, there is a monotonically decreasing trend of the maximum stress, as shown in Fig. 4(b). This arises as a constant effective bearing area is maintained, and the remaining factors influencing the maximum stress are thus crack tip bluntness and stress concentration. The combined effect of these two factors yields a decreasing trend of stress with key-hole radius. As is evident from Fig. 4, three principal factors affect the maximum stress of key-hole notched components, crack tip hole bluntness, stress concentration around the key-hole due to the finite plate size, and the reduction of the effective bearing area. Combining these three factors, we obtained the semi-analytical equation for predicting the maximum stress and validated the predictions with the FEM results.

\section{Stress analysis of Key-hole-like (U- and blunt V-) notches}

In order to further expand the application of the developed formula, we will generalize the semianalytical model (Eq. (8)) to key-hole-like notches (i.e., U- and blunt V-notches) and further examine failure criteria for notched components. In the past decade most studies on the brittle fracture of components containing key-hole-like notches were based upon the NFM extension of classical fracture mechanics, pertaining to notched engineering structures [13-16]. Various failure criteria in the field of NFM were developed using the concepts of NSIF and NFT, among others. These criteria usually involve a number of model parameters, requiring complex fitting of experimental data, motivating the use of reliable analytical models. Here we apply the semianalytical equation proposed in the Section 2 to investigate the maximum stress of U-notches and blunt V-notches shown in Fig. 5(a). 

solution for stress at round-tip notches. Engineering fracture mechanics, 149, 134-143.

First, plates containing blunt V-notches and loaded by remote tensile stress $\sigma_{\infty}$, as shown in Fig. 5(b), are studied by the FEM technique described in the preceding section. Notches with tip curvature $\rho / w=1 / 15$ and different opening angles $2 \alpha\left(0,53^{\circ}, 90^{\circ}, 113^{\circ}, 127^{\circ}\right)$ were taken into account. As a special case, the blunt V-notch with $\alpha=0$ degenerates into a U-notch, see Fig. 5(a). The contour plots of the $y$-direction normal stress of the key-hole notch, U-notch and blunt V-notches with different opening angles are shown in Fig. 5(c-h) where the specimen dimensions are $b / w=2, a / w=5 / 12$ and $\rho / w=1 / 15$. As is evident from Fig. 5, the stress distributions and maximum stresses at U-notches and V-notches with small opening angles are essentially similar to those of the key-hole notches. A close examination of Fig. 5(c) for a keyhole notch and Fig. 5(d) for a U-notch of the same hole radius and crack length shows clearly that the stress distribution and maximum stress of the U-notch can be well approximated by those of the corresponding key-hole notch. With the opening angle of the blunt V-notches increasing (Figs. 5(e-h)), the maximum stress slightly decreases.

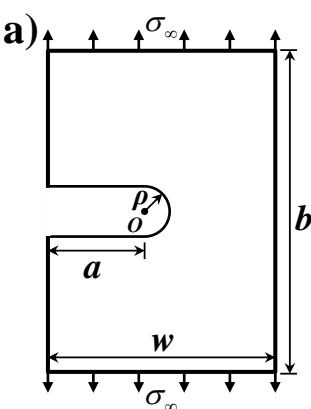

e)

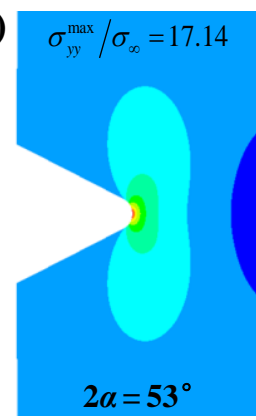

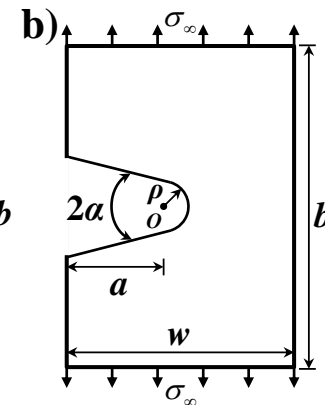

f)

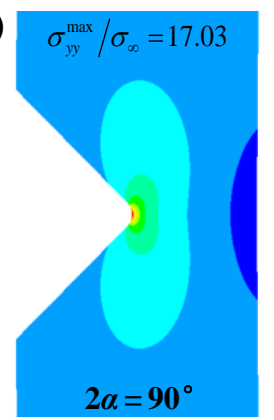

c)

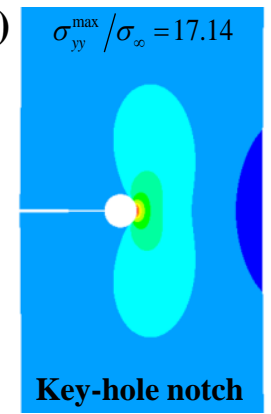

g)

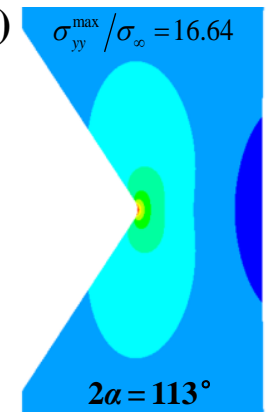

d)

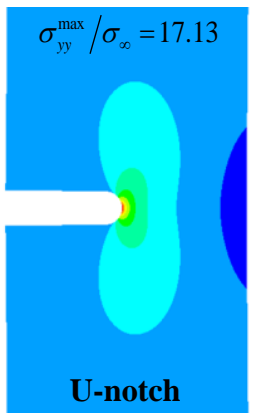

h)

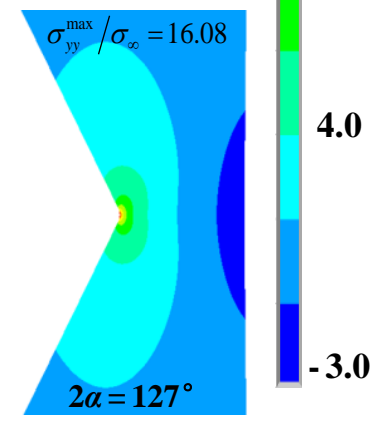

Fig. 5 Tensioned plates weakened by U-notch (a) and blunt V-notch (b). Contours of normal stress $\sigma_{y y}$ for key-hole notch (c), U-notch (d) and blunt V-notches with different opening angles (eh). The geometrical parameters are: $b / w=2, a / w=5 / 12$, and $\rho / w=1 / 15$. All the stress profiles are scaled in range of $-30 \sim 175 \mathrm{MPa}$.

In order to quantify the extent of approximation of V-notches (as shown in Fig. 5(b)) in terms of the corresponding key-hole notches (see Fig. 1(a)), a series of FEM simulations are conducted for components with blunt V-notches with various crack lengths $a(a / w=0.28$, $0.42,0.55)$, notch tip curvature $0 \leq \rho / w \leq 0.6$, and opening angles $0 \leq 2 \alpha \leq 130^{\circ}$. Contours 
Liu, M., Gan, Y., Hanaor, D. A., Liu, B., \& Chen, C. (2015). An improved semi-analytical solution for stress at round-tip notches. Engineering fracture mechanics, 149, 134-143.

of the relative difference between the maximum notch-tip stresses of V-notches and that of the corresponding key-hole notches (or U-notches) of the same notch dimensions are shown in Fig. 6 , where the horizontal and vertical axes represent the opening angle $2 \alpha$ and the relative notch tip curvature $\rho / a$, respectively. The corresponding relative crack lengths in Figs. 6(a-c) are $a / w=0.28,0.42$ and 0.55 . It can be seen that the maximum stress of V-notches can be well described by the developed semi-analytical model for key-hole notches, with the relative difference well below $5 \%$ in most of the $\alpha-\rho$ space. Only when $2 \alpha \geq 90^{\circ}$ and $\rho / a \leq 0.1$ (see the small heavily shaded regions in Fig. 6), the relative difference reaches $10 \%$ or even higher. Moreover, for notches of larger depths, an even larger regime in the $\alpha-\rho$ space is found to have relative difference less than $5 \%$.
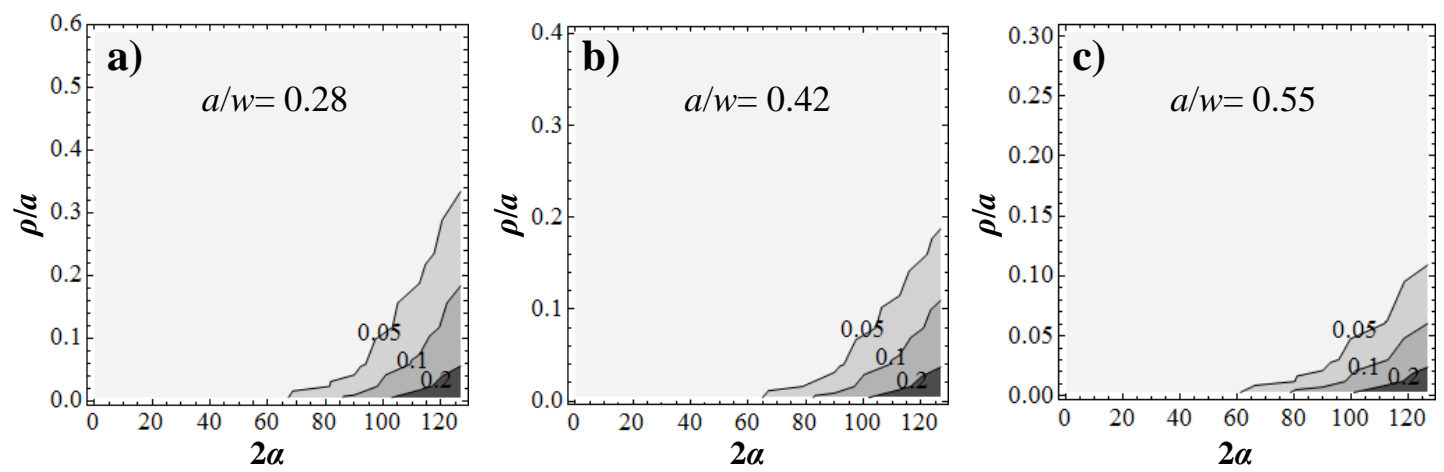

Fig. 6 The relative difference between maximum notch-tip stresses in blunt V-notches with different opening angles and the corresponding key-hole notches (or U-notches) of the same crack length $a$ and tip curvature $\rho$. The relative crack lengths are: (a) $a / w=0.28$, (b) 0.42 , and (c) 0.55 .

From Fig. 6, we can conclude that the proposed semi-analytical is applicable for the prediction of the maximum stress for a variety of notch types, including key-hole, U- and blunt V-shapes with a wide geometrical parameters (radius, notch depth and opening angle). Such a prediction of the maximum stress can be adopted to analyze the damage in notched components and establish of failure criteria, as illustrated in the following.

\section{Discussion on stress-based failure criteria for notched components}

The proposed semi-analytical solution for the maximum stress in key-hole and key-hole-like problems can be implemented in various stress-based failure criteria. We discuss its potential applications in the following two typical stress-based criteria.

\subsection{Maximum stress based failure criterion}

For brittle materials, they are often deemed to fail when the maximum tensile stress $\sigma_{y y}^{\max }$ 
(usually the normal stress at the notch tip of the cross section, as labeled in Fig. 1(b)) reaches the material's failure strength $\sigma_{f s}$ [38]. Therefore, a simple method of predicting failure strength is based on the criterion of

$$
\sigma_{y y}^{\max } \leq \sigma_{f s}
$$

This failure criterion can be applied for assessing cracks with small key-holes and notches with large holes, including U-notches and blunt V-notches [39]. Using the semi-analytical model (i.e., Eq. (8)) in conjunction with Eq. (13), one can predict the maximum admissible load of plates with notches.

\subsection{Notch tip stress field based fracture criteria}

In general, most failure criteria for notched components are based on the notch tip stress field. In these criteria, the notch tip maximum stress has been introduced into the stress field as a key parameter [21-24]. For example, in the widely used stress field solution for rounded-tip Vnotches obtained by Filippi et al. [21], the $y$-direction normal stress along the bisector for a notch with opening angle $2 \alpha$ and notch tip curvature $\rho$ is given by

$$
\sigma_{y y}=\sigma_{y y}^{\max } \cdot r^{\lambda_{1}-1} \cdot f(\alpha, \rho) \text {. }
$$

where $r$ is the polar coordinate, $\lambda_{1}$ is a function of the notch opening angle. This equation has been found to be in good agreement with finite element results in the high stress region around the notch tip [21]. Subsequently, this stress field has further been extended to plates with finite width [22].

In addition, the NSIF, which indicates the stress intensity at the notch tip, can be related to the maximum stress by the following equation

$$
K_{\mathrm{I}}^{V}=\sigma_{y y}^{\max } \cdot \sqrt{2 \pi} \cdot g(\alpha, \rho)
$$

It can be seen from Eqs. (14) and (15) that the maximum stress $\sigma_{y y}^{\max }$ is contained in the notch tip stress field based failure criteria as an unknown parameter and varies with the geometry size of the components and the load conditions. By substituting the semi-analytical equation (Eq. (8)) into Eqs. (14) and (15), the $y$-direction normal stress $\sigma_{y y}$ and NSIF $K_{\mathrm{I}}^{V}$ can be directly related to the external load $\sigma_{\infty}$.

\section{Conclusions}

In this paper, the stress distributions of notched components under mode I loading are investigated. Based upon the combined effects of three mechanisms, crack tip bluntness, stress concentration and stress equilibrium; a semi-analytical formula is proposed to predict the notch 
tip maximum stress. Due to the competition of the three aforementioned mechanisms, the maximum stress at the notch tip first decreases and then increases with increasing notch tip radius for a fixed crack length. For constant notch depth the maximum stress is found to decrease monotonically with increasing notch tip radius. An extensive comparison between the theoretical prediction of the maximum stresses and FEM results was shown to validate the new semi-analytical model.

It is further found that the stress fields of U-notches and blunt V-notches with small tip radii and opening angles are similar to those of key-hole notches. The proposed semi-analytical model can therefore be applied to predict the maximum stress at the tip of these notches and establish appropriate stress-based failure criteria. The model can also be applied to edge notched infinite plates. The presently developed approach can be extended to round-tip center notches and can further be generalized to incorporate alternative loading conditions including localized loads.

\section{Acknowledgements}

The authors are grateful for the financial support of this work by the National Natural Science Foundation of China (No. 11472149), the National Basic Research Program of China (No. 2011CB610305), and the Tsinghua University Initiative Scientific Research Program (No. 2014z22074).

\section{References}

[1] Kuang ZB. The stress field near the blunt crack tip and the fracture criterion. Eng. Fract. Mech. 1982; 16(1): 19-33.

[2] Glinka G, Newport A. Universal features of elastic notch-tip stress fields. Int. J. Fat. 1987; 9(3): 143-150.

[3] Lazzarin P, Tovo R. A unified approach to the evaluation of linear elastic stress fields in the neighborhood of cracks and notches. Int. J. Fract. 1996; 78(1): 3-19.

[4] Gomez FJ, Guinea GV, Elices M. Failure criteria for linear elastic materials with U-notches. Int. J. Fract. 2006; 141(1-2): 99-113.

[5] Torabi AR, Pirhadi E. Stress-based criteria for brittle fracture in key-hole notches under mixed mode loading. Eur. J. Mech. Solids/A 2015; 49: 1-12.

[6] Gómez FJ, Elices M, Berto F, Lazzarin P. Fracture of U-notched specimens under mixed mode: experimental results and numerical predictions. Eng. Fract. Mech. 2009; 76(2): 236249.

[7] Ayatollahi MR, Torabi AR. Brittle fracture in rounded-tip V-shaped notches. Mater. Design 2010; 31(1): 60-67.

[8] Berto F, Zappalorto M. Fictitious notch rounding concept applied to V-notches with end holes under mode I loading. Int. J. Fract. 2011; 171(1): 91-98.

[9] Torabi AR, Abedinasab SM. Brittle fracture in key-hole notches under mixed mode 
loading: Experimental study and theoretical predictions. Eng. Fract. Mech. 2015; 134: 3553.

[10] Torabi AR, Abedinasab SM. Fracture study on key-hole notches under tension: two brittle fracture criteria and notch fracture toughness measurement by the disk test. Exper. Mech. 2015; 55(2): 393-401.

[11] Torabi AR, Fakoor M, Pirhadi E. Tensile fracture in coarse-grained polycrystalline graphite weakened by a U-shaped notch. Eng. Fract. Mech. 2013; 111: 77-85.

[12] Anderson TL. Fracture mechanics: fundamentals and applications. Boca Raton: CRC press; 2005.

[13] Radaj D. State - of - the - art review on extended stress intensity factor concepts. Fat. Fract. Eng. Mater. Struct. 2014; 37(1): 1-28.

[14] Gomez FJ, Elices M, Valiente A. Cracking in PMMA containing U-shaped notches. Fat. Fract. Eng. Mater. Struct. 2000; 23: 795-803.

[15] Carpinteri A, Cornetti P, Sapora A. Brittle failures at rounded V-notches: a finite fracture mechanics approach. Int. J. Fract. 2011; 172(1): 1-8.

[16] Gomez FJ, Guinea GV, Elices M. Failure criteria for linear elastic materials with U-notches. Int. J. Fract. 2006; 141(1-2): 99-113.

[17] Erdogan F, Sih GC, On the crack extension in plates under plane loading and transverse shear. J. Basic Eng. 1963; 85(4): 519-525.

[18] Creager M, Paris PC, Elastic field equations for blunt cracks with reference to stress corrosion cracking. Int. J. Fract. Mech. 1967; 3(4): 247-252.

[19] Kullmer G, Richard HA. Influence of the root radius of crack-like notches on the fracture load of brittle components. Arch. Appl. Mech. 2006; 76(11-12): 711-723.

[20] Lazzarin P, Berto F, Ayatollahi MR. Brittle failure of inclined key-hole notches in isostatic graphite under in - plane mixed mode loading. Fat. Fract. Eng. Mater. Struct. 2013; 36(9): 942-955.

[21] Filippi S, Lazzarin P, Tovo R. Developments of some explicit formulas useful to describe elastic stress fields ahead of notches in plates. Int. J. Solids Struct. 2002; 39(17): 45434565.

[22] Filippi S, Lazzarin P. Distributions of the elastic principal stress due to notches in finite size plates and rounded bars uniaxially loaded. Int. J. Fat. 2004; 26(4): 377-391.

[23] Zappalorto M, Lazzarin P. In-plane and out-of-plane stress field solutions for V-notches with end holes. Int. J. Fract. 2011; 168(2): 167-180.

[24] Zappalorto M, Carraro PA. Stress distributions for blunt cracks and radiused slits in anisotropic plates under in-plane loadings. Int. J. Solids Struct. 2015; 56-57: 36-141.

[25] Bazant ZP. Size effect in blunt fracture: concrete, rock, metal. J. Eng. Mech. 1984; 110: 518-535.

[26] Smith E. Underpinning of a simple blunt flaw fracture initiation relation. Int. J. Fract. 2005; 131: 401-405.

[27] Leguillon D, Piat R. Fracture of porous materials-Influence of the pore size. Eng. Fract. Mech. 2008; 75: 1840-1853.

[28] Liu M, Chen C. A micromechanical analysis of the fracture properties of saturated porous media. Int. J. Solids Struct. 2015; 63:32-38. 
Liu, M., Gan, Y., Hanaor, D. A., Liu, B., \& Chen, C. (2015). An improved semi-analytical solution for stress at round-tip notches. Engineering fracture mechanics, 149, 134-143.

[29] Irwin GR. Analysis of stresses and strains near the end of a crack traversing a plate. ASME J. Appl. Mech. 1957; 24: 361-364.

[30] Fett T, Munz D. Stress intensity factors and weight functions. Vol. 1. Comput. Mech. 1997.

[31] Bazant ZP. Size effect in blunt fracture: concrete, rock, metal. J. Eng. Mech. 1984; 110(4): 518-535.

[32] Leguillon D, Piat R. Fracture of porous materials-Influence of the pore size. Eng. Fract. Mech. 2008; 75(7): 1840-1853.

[33] Torabi A R. Fracture assessment of U-notched graphite plates under tension. Int. J. Fract. 2013; 181(2): 285-292.

[34] Tada H, Paris PC, Irwin GR. The stress analysis of cracks handbook. New York: ASME press; 2000.

[35] Sih GC, Liebowitz H. Mathematical theories of brittle fracture. In Fracture (Vol. 2, pp. 67190) New York: Academic Press; 1968.

[36] Jia YJ, Shi MX, Zhao Y, Liu B. A better estimation of plastic zone size at the crack tip beyond Irwin's model. ASME J. Appl. Mech. 2013; 80(5): 051014.

[37] Heywood RB. Designing by Photoelastacity. London: Chapman and Hall press; 1952.

[38] Hertel D, Fett T, Munz D. Strength predictions for notched alumina specimens. J. Euro. Cera. Soc. 1998; 18(4): 329-338.

[39] Gómez FJ, Elices M. A fracture criterion for blunted V-notched samples. Int. J. Fract. 2004; 127(3): 239-264. 